\title{
Aperçu du programme de gestion des urgences du Laboratoire national de microbiologie
}

\author{
D Marcino ${ }^{1,2}, \mathrm{~K}$ Gordon ${ }^{1 *}$
}

\section{Résumé}

Le programme de gestion des urgences du Laboratoire national de microbiologie (LNM) a été mis au point après l'épidémie du syndrome respiratoire aigu sévère (SRAS) de 2003 afin de fournir un cadre d'intervention en cas de problème de santé publique. Il est composé de trois volets (intervention sur les lieux, continuité des opérations et soutien sur place) dont la structure de gestion est fondée sur le système de commandement en cas d'incident, qui suit les quatre phases de la gestion d'une situation d'urgence. Tous les volets du programme comprennent une formation poussée axée sur les compétences et des plans d'exercice pour le personnel. Le programme de gestion des urgences est soumis à un processus d'examen structuré et satisfait à la norme ISO 9001 de l'Organisation internationale de normalisation, ce qui assure sa qualité et son perfectionnement constant. Ce processus signifie que, moins d'une heure après la réception d'un avis d'intervention, il est possible de mettre en place un centre des opérations pouvant fonctionner à plein régime grâce à du personnel hautement qualifié et chevronné. Le LNM peut également mettre sur pied des laboratoires mobiles afin de contribuer aux efforts nationaux ou internationaux visant à lutter contre une épidémie ou un incident bioterroriste.

\section{Affiliations}

${ }^{1}$ Laboratoire national de microbiologie, Agence de la santé publique du Canada, Winnipeg (Manitoba)

${ }^{2}$ Réseau des laboratoires de santé publique du Canada

*Correspondance : kristina. gordon@canada.ca

Citation proposée : Marcino D, Gordon K. Aperçu du programme de gestion des urgences du Laboratoire national de microbiologie. Relevé des maladies transmissibles au Canada. 2018;44(5):113-7.

https://doi.org/10.14745/ccdr.v44i05a02f

Mots clés : Gestion urgences, planification des mesures d'urgence, préparation des laboratoires, laboratoire de santé publique

\section{Introduction}

Organisme de diagnostic et de recherche en matière de maladies infectieuses de calibre mondial, le Laboratoire national de microbiologie (LNM) collabore avec le Centre national des maladies animales exotiques et abrite le seul laboratoire de niveau de confinement 4 au Canada. Le LNM, qui a pour mission d'améliorer la santé humaine grâce au leadership en laboratoire, à l'excellence scientifique et à l'innovation en santé publique (1), est réputé pour travailler à la protection de la santé des Canadiens et contribuer considérablement aux efforts de la communauté internationale de la santé publique en période de crise.

La contribution du LNM à la santé publique mondiale a commencé en 2003, année où un agent pathogène inconnu voyait le jour partout dans le monde et mettait à l'épreuve la capacité du Canada à réagir à une crise de santé publique. Pendant que cette maladie se propageait à l'échelle mondiale, le personnel du LNM tentait d'identifier cet agent pathogène, plus tard baptisé le « syndrome respiratoire aigu sévère » (SRAS). D'autres employés travaillaient diligemment en vue d'élaborer un plan pour répondre à l'éclosion de la maladie. Les salles de conférence se sont transformées en centres d'opérations d'urgence improvisés, les communications se sont intensifiées et les employés ont collaboré pour offrir du soutien et des conseils aux partenaires de santé publique.
Depuis l'éclosion du SRAS, il y a 15 ans, les hauts gestionnaires du LNM ont appliqué les leçons tirées de cet épisode et des crises qui ont suivi. Ils se sont notamment faits les champions de la mise au point d'un programme évolutif de gestion des urgences qui est ancré dans une culture de gestion de la qualité. Étant donné que les épidémies franchissent librement les frontières et les continents, la communauté de la santé publique doit optimiser sa compréhension et sa connaissance de la gestion des urgences pour mieux s'y préparer.

Le présent article donne un aperçu du programme de gestion des urgences du LNM. On y traite notamment de l'intégration des quatre phases standard de la gestion des urgences, du cadre de gestion du système de commandement en cas d'incident $(\mathrm{SCl})$ et de la manière dont le programme est entretenu grâce à un cycle continu de perfectionnement et à l'appui sans réserve de la direction.

\section{Volets et équipes du programme de gestion des urgences}

Le plan de gestion des urgences comprend trois volets : intervention sur les lieux, continuité des opérations et soutien sur place. Les interventions peuvent être lancées à l'interne, au 
LNM ou sur le terrain. Elles peuvent être déclenchées par le LNM seulement ou au sein d'une équipe plus grande composée de partenaires canadiens ou internationaux. II ne sera pas question ici de la manière dont le LNM coordonne ses efforts à ceux de partenaires nationaux et internationaux.

\section{Intervention sur les lieux}

Il existe deux types d'intervention sur les lieux : I'intervention de l'équipe d'intervention d'urgence du bâtiment et celle du secteur de programme. L'équipe d'intervention d'urgence du bâtiment agit lorsque l'incident touche directement l'installation - par exemple, en cas de réception d'un colis suspect, d'incendie ou d'urgence médicale. Les secteurs de programmes se penchent sur les incidents comprenant un pathogène où le LNM peut intervenir sur les lieux en collaboration avec les gouvernements fédéral, provinciaux et territoriaux. Par exemple, la Section des virus respiratoires et de l'influenza est un secteur de programme qui est intervenu pendant la pandémie de H1N1 en 2009.

La Section des zoonoses virales, quant à elle, est intervenue pendant l'épidémie de Zika en 2016.

Les laboratoires mobiles du LNM sont chargés de répondre aux urgences sur le terrain si un agent pathogène ou biologique est en cause. Le LNM comprend deux groupes particuliers posant des diagnostics sur le terrain : l'équipe de diagnostic des pathogènes spéciaux qui intervient en cas d'éclosions de maladie (p. ex. la flambée du virus Ebola en 2014) et l'équipe d'intervention d'urgence microbiologique, qui vise d'abord à orchestrer les interventions en cas de bioterrorisme. Ces deux groupes sont également déployés de façon proactive. Ainsi, l'équipe d'intervention d'urgence microbiologique peut intervenir dans le cadre d'événements planifiés : par exemple, les Jeux olympiques de Vancouver en 2010 ou le prochain Sommet du G7. Par ailleurs, on peut dépêcher l'équipe de diagnostic des pathogènes spéciaux afin d'aider un partenaire extérieur à se préparer à une éclosion, à renforcer ses capacités avant l'éclosion ou à mettre sur pied un programme d'intervention. C'est ce qu'elle a fait au centre de contrôle des maladies du Nigéria, afin de lutter contre la fièvre de Lassa cette année.

\section{Continuité des opérations}

Le volet du programme de gestion des urgences relatif à la continuité des opérations comprend des fonctions avancées de planification visant à s'assurer que le LNM peut poursuivre ses activités essentielles en cas d'urgence ou lorsque les installations, l'équipement ou le personnel sont inaccessibles. Dans le cadre des activités de planification de la continuité des opérations, il faut, par exemple, prévoir des installations de rechange et des programmes de dotation accélérée permettant d'offrir une formation polyvalente aux travailleurs de laboratoire, de manière à pouvoir remplacer provisoirement les employés qui sont appelés à répondre à des urgences. En activant ces plans pendant une situation d'urgence, le LNM peut continuer à offrir ses services courants à ses clients.

\section{Soutien sur place}

Le volet du soutien sur place du programme de gestion des urgences correspond à l'activation du centre des opérations du LNM, qui coordonne et soutient une intervention sur les lieux ou veille à la continuité des opérations, ou les deux. Le centre des opérations est un pôle de coordination essentiel permettant d'intervenir de manière globale et concertée et de relâcher la pression exercée sur l'équipe responsable de l'intervention. Le personnel affecté au soutien sur place réalise une série de tâches. Il doit, par exemple, s'assurer que le matériel logistique et les documents de voyage sont disponibles, que les dispositions de voyage ont été priseset que le personnel sur place a été vacciné comme il se doit pour pouvoir participer sans danger à l'intervention.

\section{Phases de la gestion des urgences}

Tous les volets du programme de gestion des urgences du LNM reposent sur les quatre phases standard de la gestion des urgences, à savoir l'atténuation, la préparation, l'intervention et le rétablissement. Ces phases doivent être appréhendées comme un cycle continu, chaque phase reposant sur la dernière et jetant les bases de la prochaine. Cette approche permet de créer un environnement opérationnel tenant quotidiennement compte de la gestion des urgences, ce qui, en soi, contribue à atténuer I'incidence éventuelle de situations urgentes ultérieures.

Le gouvernement du Canada définit comme suit les quatre phases de la gestion des urgences:

- Atténuation : Mesures prises pour atténuer l'incidence des catastrophes afin de protéger la vie, les biens et l'environnement, et pour réduire les perturbations économiques.

- Préparation : Mesures prises avant une catastrophe ou une situation d'urgence afin d'être prêt à y répondre et à en gérer les conséquences.

- Intervention : Mesures prises pendant ou immédiatement avant ou après une catastrophe pour en gérer les conséquences et minimiser la souffrance et les pertes qui y sont associées.

- Rétablissement : Mesures prises pour restaurer ou rétablir les conditions de vie du milieu à un niveau acceptable après une catastrophe (2).

Les volets du programme de gestion des urgences du LNM correspondent donc à chacune de ces phases. De plus, chaque équipe d'intervention du LNM a intégré ces phases à sa stratégie opérationnelle. Cette approche sert au fonctionnement du LNM de trois façons. Premièrement, elle a permis de dresser une liste d'employés très qualifiés et chevronnés qui sont prêts à exécuter les plans d'intervention. Deuxièmement, les équipes d'intervention ont eu carte blanche pour préparer l'inventaire de l'équipement requis, ainsi que des plans et des programmes d'entretien. Troisièmement, elle a permis de bâtir une main-d'œuvre désireuse d'appliquer les leçons tirées lors de chaque crise. Toutes ces mesures permettent d'accroître la résistance des ressources humaines qui devront composer avec d'autres crises.

\section{Système de commandement en cas d'incident}

Le $\mathrm{SCl}$ est un cadre de gestion qui est utilisé par les premiers répondants depuis les années 1970. Ce système universel éprouvé facilite la coordination intragouvernementale et 
intergouvernementale de la gestion des événements notamment grâce à des rôles et des responsabilités qui ont été établis conjointement, d'une structure organisationnelle évolutive et de processus normalisés (3). Le $\mathrm{SCl}$ est intégré à tous les volets du programme de gestion des urgences du LNM. Grâce à ce système de gestion normalisé et évolutif, les répondants du LNM peuvent rapidement adopter des protocoles leur permettant de réagir proportionnellement à l'envergure d'une situation et d'intégrer rapidement leurs opérations lorsqu'ils travaillent avec des premiers répondants et avec des partenaires canadiens ou internationaux.

La structure organisationnelle du SCl du centre des opérations est très robuste (figure 1). L'un des aspects importants de cette structure est qu'elle peut être adaptée à chaque situation. Par exemple, pendant des crises majeures - la pandémie de H1N1 en 2009 - tout le personnel a pu être mobilisé 24 heures sur 24 , sept jours sur sept. Inversement, pendant des crises plus circonscrites (comme les mobilisations récentes de l'équipe de diagnostic des pathogènes spéciaux en Sierra Leone pour le projet de biobanque de virus Ebola), le personnel du centre des opérations a été réduit pour ne comprendre que les postes essentiels, comme le directeur, l'agent de liaison et le chef de la logistique.

\section{Autres aspects}

Outre les quatre volets de la gestion des urgences et le $\mathrm{SCl}$, l'efficacité du programme de gestion des urgences est assurée par un effectif se consacrant à la gestion des urgences, par le contrôle de la qualité, un programme de formation et d'exercice, et un soutien solide et continu de la part de la direction.

\section{Personnel de gestion des urgences}

Le centre des opérations compte quatre employés permanents à temps plein, y compris un gestionnaire des urgences accrédité, dont les fonctions sont essentiellement de veiller à ce que le LNM soit prêt à répondre à toute situation d'urgence. Le personnel fournit une gamme de services, y compris de la formation et des exercices. II met au point des inventaires pour le système de commandement en cas d'incident et veille à ce que les appareils électroniques et autres biens soient disponibles et en bon état de fonctionnement.

\section{Programmes de formation et d'exercices}

Tous les volets du programme comprennent une formation poussée axée sur les compétences et des plans d'exercice pour le personnel. Avec le soutien et l'autorité de la haute direction, depuis 2009, 625 personnes ont suivi une formation et plus de $60 \%$ d'entre elles ont été sélectionnées pour approfondir leur formation - et certaines ont obtenu une certification en gestion des urgences. La liste des personnes ayant participé à la formation et aux exercices est consignée dans le système de gestion de l'information du LNM. Grâce à ce bassin de personnes qualifiées en gestion des urgences, nous pouvons toujours compter sur des spécialistes hautement qualifiés et chevronnés susceptibles de répondre à tous les aspects du programme de gestion des urgences pendant de longues

Figure 1 : Structure organisationnelle du centre des opérations du Laboratoire national de microbiologie

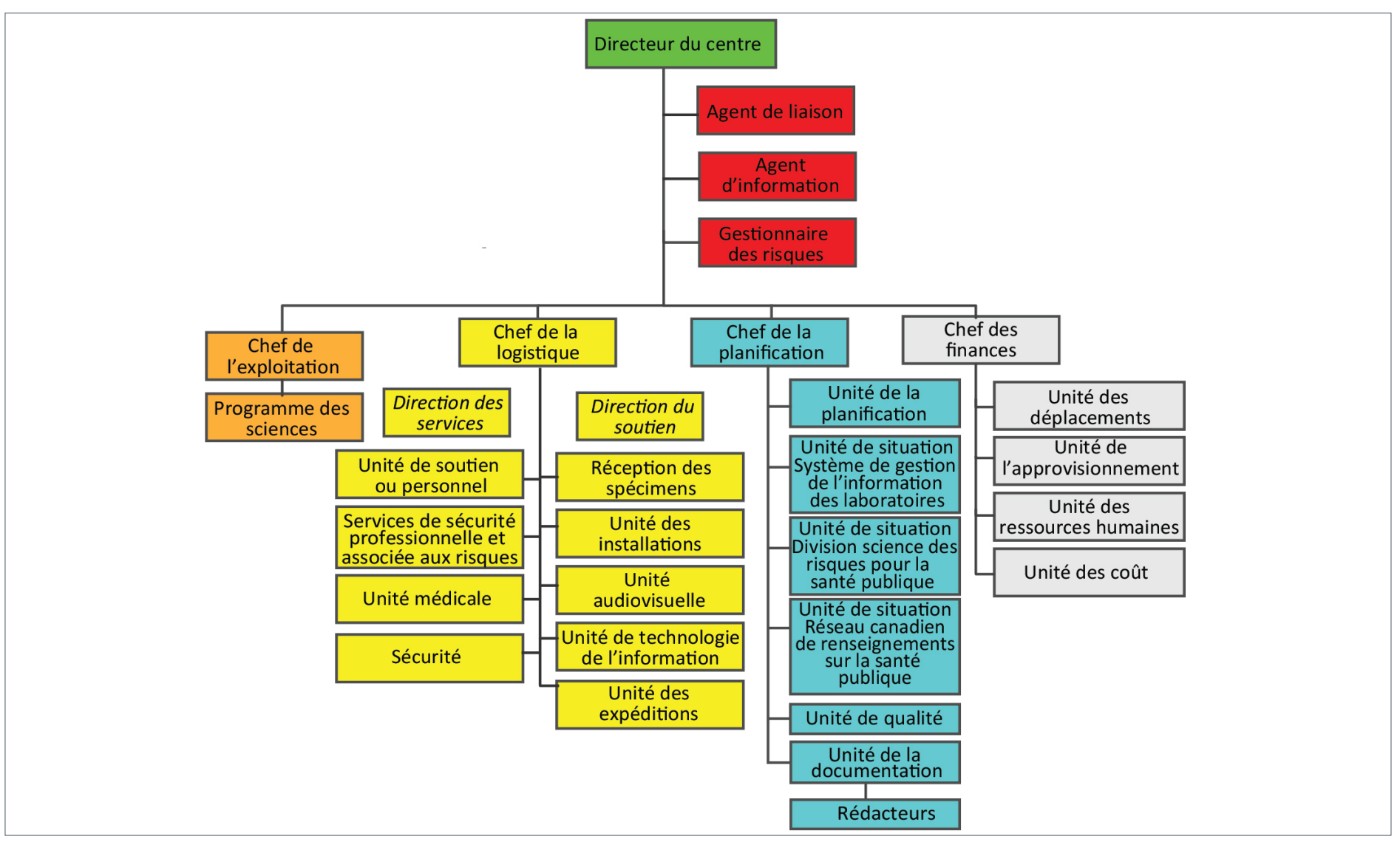


périodes et à plusieurs reprises, ce qui est essentiel au bon fonctionnement du programme.

\section{Contrôle de la qualité et perfectionnement continu}

La culture entourant le programme de gestion des urgences valorise l'excellence et le perfectionnement continu. En 2012, le programme a obtenu la certification ISO 9001 de l'Organisation internationale de normalisation. L'ISO 9001 est la norme internationale établissant les exigences auxquelles un système de gestion doit répondre pour atteindre un certain niveau de qualité (4). Le Bureau de la qualité du LNM veille à ce que le système demeure conforme aux exigences de la certification ISO 9001. Ainsi, le programme de gestion des urgences fonctionne efficacement selon des objectifs précis, ce qui montre aux clients et aux intervenants que leurs exigences sont respectées conformément aux lois fédérales et provinciales. Les exigences de la certification ISO 9001 sont intégrées au programme et contribuent à sa cohérence, en plus de favoriser la satisfaction du client.

L'une des exigences importantes de la stratégie de perfectionnement continu et de la certification ISO consiste à appliquer les leçons apprises au fil des interventions d'urgence. Pour faciliter ceci, le LNM mène des discussions "à chaud" immédiatement après chaque intervention. Cet exercice vise à rassembler toutes les personnes qui ont participé à une intervention afin de faire le point sur les forces, les faiblesses et les défis de l'intervention, et ce, dans l'optique de cerner et de documenter les possibilités d'amélioration dans une analyse après action. Les analyses après action font partie intégrante de la stratégie de perfectionnement continu du programme, car elles contiennent des recommandations hiérarchisées en vue d'améliorer le programme. Ces recommandations sont passées en revue et approuvées par la haute direction. Elles sont ensuite confiées à des employés du centre des opérations ou des secteurs de programmes du LNM. Les recommandations sont gérées par le système de gestion de l'information des laboratoires pour veiller à ce qu'elles soient mises en œuvre correctement et intégrées aux interventions ultérieures.

\section{Soutien sans réserve de la direction}

Le succès du programme de gestion des urgences est profondément enraciné dans l'engagement à long terme de la haute direction. Le dévouement de cette dernière a permis de mettre au point un programme qui comble les lacunes mises en évidence par la crise du SRAS et auquel les employés adhèrent sans réserve. L'organisation a donc adopté une philosophie en vertu de laquelle la vocation du LNM n'est pas seulement de contribuer à la santé publique, mais également aux interventions d'urgence nationales et internationales dans le domaine de la santé publique.

Trois des plus importantes décisions prises par la haute direction au début de l'élaboration du programme de gestion des urgences étaient la mise en place d'un centre des opérations physique, l'embauche de spécialistes chevronnés pour mettre au point le programme et exploiter le centre, ainsi qu'une formation de base obligatoire au $\mathrm{SCl}$ et au centre d'opération d'urgence. Le centre des opérations physique est important, car il garantit que les biens nécessaires pour orchestrer une intervention sont en place. De cette manière, l'intervention en cas d'urgence peut être lancée rapidement et dans les meilleures conditions. Moins d'une heure après avoir reçu un avis, le centre des opérations peut être doté d'une équipe homogène composée de personnes qualifiées.

Pour mettre au point le programme de gestion des urgences, le LNM a pourvu des postes permanents et à temps plein. L'embauche de ces spécialistes a été bénéfique à plusieurs égards. D'abord, parce qu'ils connaissent en profondeur le domaine de la gestion des urgences. Il arrive souvent qu'une organisation attache le rôle de gestionnaire des urgences à la description d'un poste existant, mais qui n'a rien à voir avec les urgences - cette fonction est donc assumée quand le temps et les connaissances du titulaire le permettent et le programme de gestion des urgences ne gagne jamais l'importance ou l'appui dont il aurait besoin pour s'épanouir. Pour gérer les quatre phases de la gestion des urgences, il est crucial de pouvoir compter sur un personnel qualifié et dévoué, qui a l'appui des responsables de l'organisation. Le personnel du centre des opérations dirige les activités de préparation et d'atténuation de l'organisation, par exemple, les programmes de formation, les exercices et l'élaboration des plans d'intervention. Pendant I'intervention, les responsables du centre des opérations s'assurent de pouvoir compter sur tout le personnel nécessaire et veillent à ce que les protocoles et les mesures visant la qualité soient respectés. Ils orchestrent également les activités de rétablissement - par exemple l'analyse après action et le suivi et la mise en œuvre des leçons tirées.

L'initiation obligatoire de tous les employés à la gestion des urgences s'avère également très précieuse pour obtenir l'appui du personnel. On s'assure ainsi que tout le personnel comprend au moins les fonctions d'urgence essentielles du LNM. La formation est donnée au LNM, ce qui a permis de diminuer les dépenses et les pertes de temps.

\section{Conclusion}

Depuis la crise du SRAS, la gestion des urgences a évolué au LNM. II ne s'agit plus d'un effort ponctuel, mais d'une fonction importante intégrée aux opérations quotidiennes et à la philosophie organisationnelle. La mise en œuvre de ce programme a permis au LNM de devenir un chef de file dans le domaine et de contribuer au développement de programmes semblables dans des laboratoires partout au Canada et dans le monde. Dans ce contexte, il a tissé des relations précieuses sur lesquelles il peut immédiatement compter en cas de situation d'urgence, notamment aux fins de coordination. Si la tâche de mettre au point un programme de gestion des urgences peut sembler énorme, les bénéfices sont immenses et nécessaires pour veiller à la santé des Canadiens et des citoyens du monde. 


\section{Déclaration des auteurs}

D. M. - Conceptualisation, rédaction de la première ébauche, examen et révision

K. G. - Conceptualisation, examen et révision

\section{Conflit d'intérêt}

Aucun.

\section{Remerciements}

Les auteurs aimeraient remercier Kelly Keith (Laboratoire national de microbiologie) et Ted Kuschak (Laboratoire national de microbiologie, Réseau des laboratoires de santé publique du Canada) pour leur contribution à cet article.

\section{Références}

1. Agence de la santé publique du Canada, Rapport des accomplissements - Excellence, innovation, leadership. Ottawa (Ontario), Agence de la santé publique du Canada; 2008. http://publications.gc.ca/collections/collection_2012/ aspc-phac/HP60-2-2008-fra.pdf

2. Bureau de la traduction. Vocabulaire de la gestion des urgences. Bulletin de terminologie; 281. [Consulté le 27 mars 2018]. http://publications.gc.ca/site/fra/9.588094/ publication.html

3. Incident Command System Resource Center. Training.fema. gov. 2018 [Consulté le 21 mars 2018]. https://training.fema. gov/emiweb/is/icsresource/

4. ISO $9001: 2015$ - What is the 9001 Version 2015 Standard?. Milwaukee (Wisconsin): American Society for Quality; 2018 [Consulté le 5 mars 2018]. http://asq.org/learn-aboutquality/iso-9000/iso-9001-2015/ 